\title{
Fertility Histories: With and Without Restrictions - An Analysis of PLM Data*
}

\author{
Z UBED A KHAN*
}

During the last decade, a large number of countries participated in the World Fertility Survey but few of them collected fertility histories that were not partially restricted. In a majority of the cases information on the duration of breast-feeding and contraceptive use was restricted to the last closed and the open intervals only. These restrictions on the fertility histories have raised many questions about the possibility of sample selection bias in the results. A number of researchers in the developed countries have used these surveys for analyzing the effects of breastfeeding and contraception on the length of birth intervals. They have acknowledged the possibility of a bias in the results and have taken measures to minimize these potential biases.

In this paper we will initially discuss the ways in which biased histories produce a biased sample of births. Later we will evaluate the effects of the restrictions by using the fertility data from the Population Labour Force and Migration (PLM) Survey. This data contains detailed reproductive histories of 9416 currently married women having 38,746 children selected from 11,000 households sampled in the PLM survey.

There are two distinct issues in this regard. The first is the extent to which the selection of the last closed and open interval leads to biased estimates of the duration of breast-feeding and the levels of contraceptive use.

The second is whether such restrictions bias the findings regarding the structure of relationships between the variables of interest.

The selective nature of using last closed and open birth intervals can best be seen by considering cohorts of birth intervals begun various years preceding the interview. Figure 1 shows the percentage of births in each year that began the last closed or open birth intervals.

*The author is Research Demographer at the Pakistan Institute of Development Economics, Islamabad. This is an abridged version of the paper presented at the Fifth Annual General Meeting of the Pakistan Society of Development Economists. 
This yields 96 logistic regression runs. ${ }^{1}$

Taking the estimates based on intervals begun 2-12 years before the interview as a comparison point, we then established a confidence interval around the betas from this restricted model that is equal to plus or minus twice the standard error of the betas. We examined the corresponding betas from the other models to see whether they fell within this interval or not.

Table 1 summarises the results. We have taken socio-economic and intermediate variables separately as well as combined in the four time periods under study.

\section{Table 1}

Percentage of $B_{s}$ Falling within the Confidence Interval for $B_{s}$ in the 2-12 Years Unrestric ted Model, by Type of Restrictions, Type of Varia bles, and Number of Years Preceding the Survey*

Type of Variables

and Restrictions

Number of Years Preceding the Survey

\section{Socio-economic Variables}

$\begin{array}{lrrrr}\text { WFS Restriction } & 65 & 48 & 50 & 25 \\ \text { No Restriction } & 100 & 96 & 85 & 81\end{array}$

Intermediate Variables

WFS Restriction

82

57

49

No Restriction

100

94

94

\section{All Variables}

WFS Restriction

No Restriction

100

95

90 80

*Confidence interval equal to plus or minus twice the standard error of beta for analogous no - restriction 2-12 model.

One thing is obvious, that the longer the period preceding the survey in the unrestricted sample the higher the proportion of unbiased results. In this table 85 to 96 percent of the results are unbiased. But when we restricted the fertility history to the last closed and open interval the results are biased with only 25 to 57 percent of the betas lying within the confidence interval, the rest of the results are significantly different. The estimates for the levels of the duration of breastfeeding and contraceptive use are biased upto 75 percent.

${ }^{1}$ The detailed results can be had from the author on request. 